\title{
Catalytic Asymmetric Addition of Ethoxyvinyl- zinc to Aldehydes: Two-Carbon Homologation
}

Metal-Mediated Synthesis

Key Words

asymmetric synthesis

organozinc addition

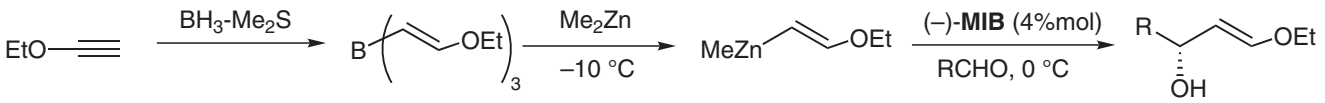

$$
\begin{aligned}
& \longrightarrow \text { 拦TBS } \\
& \text { (-)-MIB = }
\end{aligned}
$$

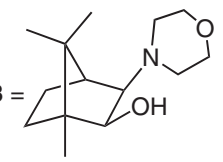

Significance: A novel catalytic non-aldol approach to $\beta$-hydroxyaldehydes overcomes many problems usually appearing during the synthesis of such systems: need for very low temperatures for asymmetric allylation or a difficult to perform asymmetric acetate aldol. This reaction requires only low loading of the chiral catalyst and is operationally simple to run.
Comment: Construction of 'acetate aldol' often presents a synthetic problem. Usually applied asymmetric allylation is not always reliable and is difficult to scale-up. Recently developed new oxazalidinones are expensive and the whole sequence is rather laborious. The powerful tool of asymmetric addition of organozinc compounds to aldehydes fits here fine and allows a new and practical solution of this important synthetic problem. 